\title{
Therapeutic Strategy for the Prevention of Pseudorabies Virus Infection in C57BL/6 Mice by 3D8 scFv with Intrinsic Nuclease Activity
}

\author{
Gunsup Lee ${ }^{1,2}$, SeungChan Cho', Phuong Mai Hoang', Dongjun Kim', Yongjun Lee', Eui-Joon Kil', \\ Sung-June Byun ${ }^{3}$, Taek-Kyun Lee ${ }^{4}$, Dae-Hyun Kim², Sunghan Kim ${ }^{5, *}$, and Sukchan Lee ${ }^{1, *}$
}

\begin{abstract}
3D8 single chain variable fragment (scFv) is a recombinant monoclonal antibody with nuclease activity that was originally isolated from autoimmune-prone MRL mice. In a previous study, we analyzed the nuclease activity of 3D8 scFv and determined that a HeLa cell line expressing 3D8 scFv conferred resistance to herpes simplex virus type 1 (HSV1) and pseudorabies virus (PRV). In this study, we demonstrate that 3D8 scFv could be delivered to target tissues and cells where it exerted a therapeutic effect against PRV. $P R V$ was inoculated via intramuscular injection, and 3D8 scFv was injected intraperitoneally. The observed therapeutic effect of 3D8 scFV against PRV was also supported by results from quantitative reverse transcription polymerase chain reaction, southern hybridization, and immunohistochemical assays. Intraperitoneal injection of 5 and $10 \mu \mathrm{g}$ 3D8 scFv resulted in no detectable toxicity. The survival rate in C57BL/6 mice was 9\% after intramuscular injection of $10 L D_{50} P R V$. In contrast, the 3D8 scFv-injected C57BL/6 mice showed survival rates of $57 \%(5 \mu \mathrm{g})$ and $47 \%(10 \mu \mathrm{g})$. The results indicate that 3D8 scFv could be utilized as an effective antiviral agent in several animal models.
\end{abstract}

\section{INTRODUCTION}

The pseudorabies virus (PRV) has a broad host range, infecting most mammals and some avian species. PRV infection frequent-

${ }^{1}$ Department of Genetic Engineering, Sungkyunkwan University, Suwon 440-746, Korea, ${ }^{2}$ Fruit Research Division, National Institute of Horticultural and Herbal Science, Rural Development Administration, Suwon 440-706, Korea, ${ }^{3}$ Animal Biotechnology Division, National Institute of Animal Science, Rural Development Administration, Suwon 441-706, Korea, ${ }^{4}$ South Sea Environment Research Department, Korea Institute of Ocean Science and Technology, Geoje 656-834, Korea, ${ }^{5}$ Department of Plant Science, Research Institute of Agriculture and Life Sciences, and Plant Genomics and Breeding Institute, Seoul National University, Seoul 151-921, Korea

*Correspondence: cell4u@skku.edu (SL); shkim4328@snu.ac.kr (SK)

Received 18 March, 2015; revised 9 June, 2015; accepted 22 June, 2015; published online 7 August, 2015

Keywords: 3D8 scFv, chemokine, mouse, nuclease activity, PRV, therapeutic effects ly proves fatal in young piglets and other susceptible species, and infected animals tend to die from central nervous system disorders (Enquist, 1999; Field and Hill, 1974; McCracken et al., 1973; Pensaert and Kluge, 1989). Infection in the natural host is characterized by epidermal lesions, usually on the mucosal surface, with viral spread to the nervous system and the establishment of latent neuronal infections (Ono et al., 2004). As the PRV has inflicted particularly heavy damage on swine livestock farming and the husbandry industry, research into vaccines against PRV has progressed relatively rapidly, but only a few antiviral agents against the virus have been developed thus far (Stegeman et al., 1997). As a novel approach, the DNA vaccine method has been employed, and this method elicits an enhanced general immune response in livestock including swine, in which the levels of interleukin-4 and interferon-gamma increase markedly (Dory et al., 2005; Gerdts et al., 1997; Hong et al., 2002). Several approaches using antiviral agents for DNA replication inhibitors or viral reverse transcriptase inhibitors have also been used in PRV containment efforts. Although generally cost-effective, these antiviral reagents work only in a limited viral spectrum, and their efficacies are generally more prone to viral mutation (Ferraris and Lina, 2008; Morfin and Thouvenot, 2003).

Through collaboration with several research groups, we have developed the 3D8 single chain variable fragment (scFv) catalytic antibody fragment, which exhibits non-specific nuclease activity on both DNA and RNA (Kim et al., 2006). Importantly, 3D8 scFv exhibits antiviral effects against herpes simplex virus (HSV), pseudorabies virus (PRV), classical swine fever virus (CSFV), murine norovirus (MNV), 2 geminiviruses, 5 tobamoviruses and cucumovirus (Hoang et al., 2014; Jun et al., 2010; Lee et al., 2013a; 2013b; 2014). We also observed caveolae-mediated endocytosis, by which the exogenously treated 3D8 scFv protein $(10 \mu \mathrm{M})$ was internalized and detected in the cell within $2 \mathrm{~h}$ and eventually in the nucleus after an extended observation period (Jang et al., 2009).

In a continued effort to develop and refine an effective antiviral agent based on 3D8 scFv, we evaluated the antiviral effects of intraperitoneally (i.p.) injected 3D8 scFv in PRV-infected C57BL/6 mice. The results of our biochemical and cytological analyses demonstrated that 3D8 scFv could be utilized as an effective antiviral agent. Our study also demonstrates that 3D8 scFv can be delivered to target tissues via i.p. injection, and we determined the threshold dosage of the injection that does not 


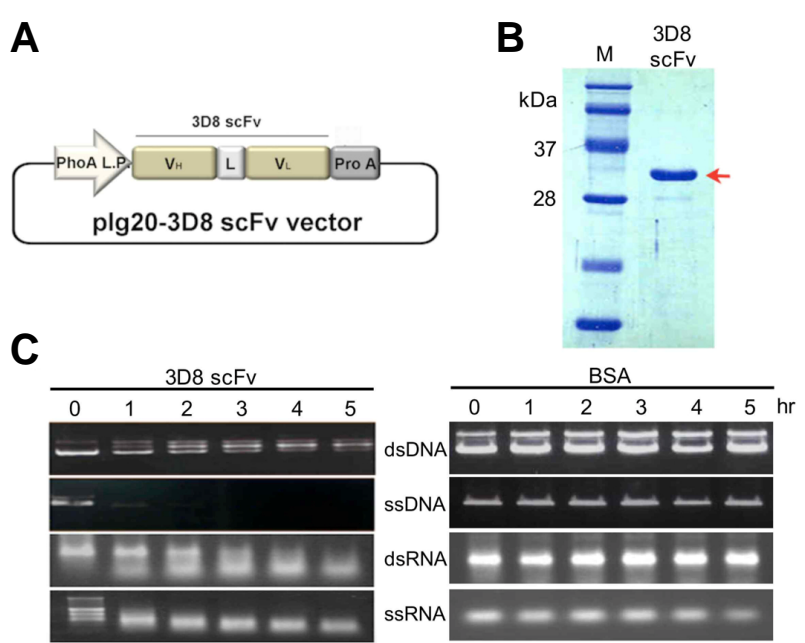

Fig. 1. Purification and catalytic activity test of $3 D 8$ single chain variable fragment (scFv) protein. (A) The plg20-3D8 scFv vector encodes a secretion signal peptide of bacterial alkaline phosphate (PhoA L.P), heavy chain variable region $\left(\mathrm{V}_{\mathrm{H}}\right)$ and light chain variable region $\left(V_{\mathrm{L}}\right)$ of the 3D8 ScFv antibody, thrombin cleavage site, and protein $A$ of Staphylococcus aureus under control of the T7 promoter. The $V_{H}$ and $V_{L}$ chains are joined by a flexible peptide linker $(\mathrm{GGGGS})_{3}$. (B) Sodium dodecyl sulfate-polyacrylamide gel electrophoresis was performed on $10 \%$ polyacrylamide gel to identify $3 \mathrm{D} 8 \mathrm{scFv}$ and stained with Coomassie Blue. The arrow is the 3D8 scFv protein (32 $\mathrm{kDa})$. Lane M: molecular weight marker. (C) The BSA and purified endotoxin-free 3D8 scFv protein $(0.2 \mu \mathrm{g})$ was mixed with $0.25 \mu \mathrm{g}$ of substrate (ssDNA, dsDNA, ssRNA, and dsRNA). A catalytic activity assay was conducted dependent on reaction time $(0,1,2,3,4$, and $5 \mathrm{~h})$. Collected samples showed a degradation pattern following agarose gel electrophoresis.

\section{cause toxicity.}

\section{MATERIALS AND METHODS}

\section{Cells and viruses}

Rat C6 glioma and B16F10 mouse melanoma cells were maintained in DMEM media supplemented with $10 \%$ fetal bovine serum (Hyclone, USA), $100 \mathrm{U} / \mathrm{ml}$ of penicillin-streptomycin (Hyclone), and non-essential amino acids (Sigma, USA), at $37^{\circ} \mathrm{C}$ in a $5 \% \mathrm{CO}_{2}$ atmosphere. The PRV-YS strain was obtained from the National Veterinary Research and Quarantine Service (NVRQS), Korea (Yoon et al., 2008). The C6 and B16F10 cell cultures were infected with PRV at a multiplicity of infection of 0.1 , and then incubated for $1 \mathrm{~h}$ in a humidified $\mathrm{CO}_{2}$ incubator at $37^{\circ} \mathrm{C}$.

\section{Mice}

Eight-week-old female C57BL/6 mice (Dae Han Bio Link Co., Ltd., Korea), weighing 18-22 g each, were maintained under standard laboratory conditions (tap water, constant room temperature of $22^{\circ} \mathrm{C}$ ). All animal procedures performed in this study (permit number: KU15006) were reviewed, approved, and supervised by the Institutional Animal Care and Use Committee of Konkuk university.

\section{Antibodies}

Anti-PRV gD monoclonal antibody was purchased from Jeno Biotech Inc. (Korea). Preparation of the anti-3D8 scFv polyc-
Ional antibody has been described previously (Lee et al., 2013a).

\section{Expression and purification of the 3D8 scFv protein}

E. coli strain BL21 DE3 ( $p L y s E$ ) cells containing plg20-3D8 were grown in $1 \mathrm{~L}$ of LB broth with $100 \mu \mathrm{g} / \mathrm{ml}$ ampicillin and 20 $\mu \mathrm{g} / \mathrm{ml}$ chloramphenicol at $37^{\circ} \mathrm{C}$ to express the recombinant 3D8 scFv protein (Fig. 1A). When the $A_{600}$ of the culture reached 0.8 , IPTG $(0.5 \mathrm{mM})$ was added to induce protein expression. The culture supernatant was obtained by centrifugation at $10,000 \times$ $g$ for $10 \mathrm{~min}$ at $4^{\circ} \mathrm{C}$ and filtered through a $0.45-\mathrm{nm}$ membrane. The 3D8 scFv protein was purified from the filtered supernatant using an IgG-Sepharose column (Amersham Pharmacia, USA). The column was washed with 20 bed volumes of PBS and then with two volumes of $5 \mathrm{mM}$ ammonium acetate $(\mathrm{pH} 5.0)$. The 3D8 scFv protein was eluted with $0.1 \mathrm{M}$ acetic acid $(\mathrm{pH} 3.4)$ in fractions of $1.5 \mathrm{ml}$ each. The eluate in the fractions was neutralized to $\mathrm{pH} 7.0$ with 0.1 volume of $1 \mathrm{M}$ Tris-base $(\mathrm{pH}$ 9.5). The 3D8 scFv protein was analyzed by $10 \%$ sodium dodecyl sulfate-polyacrylamide gel electrophoresis under reducing conditions. Then, endotoxin contents were determined by Limulus Amebocyte Lysate (LAL) (PYROGENTTM 25 single tests $0.125 \mathrm{EU} / \mathrm{ml}$ sensitivity, Lonza, Switzerland). The LAL assay was performed in pyrogen-free tubes which $0.1 \mathrm{ml}$ of 3D8 ScFv protein (amount range from $2.5 \mathrm{ug}$ to $100 \mathrm{ug}$ ) and $\mathrm{LAL}$ reagent were added. After $1 \mathrm{~h}$ incubation at $37^{\circ} \mathrm{C}$, the tubes were observed by vertical inversion whether a stable solid clot was present or not. The visible solid clot was not observed in test tubes which 3D8 scFv protein added (The values of 3D8 scFv endotoxicity was $<0.125 \mathrm{EU} \mathrm{ml}^{-1}$ ).

\section{ssDNA and dsDNA catalytic activity test with the scFv protein}

The assay reaction was performed in assay buffer $(20 \mathrm{mM}$ Tris$\mathrm{HCl}, \mathrm{pH}$ 8.0, $50 \mathrm{mM} \mathrm{NaCl}, 5 \mathrm{mM} \mathrm{MgCl}$ ). The DNA and RNA binding test was performed dependent on reaction time. DNA and RNA (0.25 $\mu \mathrm{g}$ each) were mixed with $0.2 \mu \mathrm{g}$ purified scFv protein and BSA, and samples were collected after 0, 1, 2, 3, 4, and $5 \mathrm{~h}$. Agarose gel electrophoresis was performed in $1.0 \%$ agarose gel and stained with ethidium bromide.

\section{Immunocytochemistry}

Confocal microscopy was conducted as described previously (Jang et al., 2009). Cells on coverslips were washed in PBS and fixed for $10 \mathrm{~min}$ in $4 \%$ paraformaldehyde in PBS at room temperature. The cells were permeabilized with Perm-buffer ( $1 \%$ BSA, $0.1 \%$ saponin, and $0.1 \%$ sodium azide in PBS) for $10 \mathrm{~min}$ at room temperature (RT). After $1 \mathrm{~h}$ of blocking with $3 \%$ bovine serum albumin in PBS, 3D8 scFv-treated cells were incubated with rabbit anti-3D8 scFv antibody, followed by incubation with TRITC-anti-rabbit Ig. Nuclei were stained with DAPI during the last $10 \mathrm{~min}$ of incubation at RT. Cells on coverslips were mounted in Vectashield anti-fade mounting medium (Vector Labs, USA) and observed with a Zeiss LSM 510 laser confocal microscope (Carl Zeiss, Geramny) followed by analysis with Carl Zeiss LSM imaging software.

\section{FITC labeling of the 3D8 scFv protein}

FITC labeling of 3D8 scFv was conducted using an AnaTagTM 5-FITC microscale protein labeling kit (Anaspec, USA) according to the manufacturer's instructions. The reaction mixture was labeled with 5-FITC at room temperature for $1 \mathrm{~h}$ followed by purification with a spin column (Banks and Paquette, 1995; Brinkley, 1992). C57BL/6 mice were injected i.p. at $10 \mu \mathrm{g} / 20 \mathrm{~g}$ 
(per mouse) with 3D8 scFv-FITC. The organs (liver, muscle, lung, and brain) of each mouse were collected at $0,20,40,60$, 120 , and $180 \mathrm{~min}$. Total proteins were prepared using Pro-prep solution (Intron, Korea) and measured at $494 \mathrm{~nm}$ via fluorometer (Hybrid Multi-Mode Microplate Reader, BioTeck Inst. Inc., USA).

\section{Grouping and viability test}

The C57BL/6 mice were divided into seven groups (M, A-F; 21 mice in each group). Group M was a PBS-injected mock group. Groups A-C were infected via intramuscular (i.m.) injection with strain PRV-YS at the $L D_{50}$. After PRV infection, groups A-C were treated i.p. with PBS, $5 \mu \mathrm{g}$ 3D8 scFv, and $10 \mu \mathrm{g}$ 3D8 scFv, respectively. Groups D-F were infected i.m. with the PRV strain at $10 \times$ the $L D_{50}$. Following PRV infection, groups D-F were treated i.p. with PBS, $5 \mu \mathrm{g}$ 3D8 scFv, and $10 \mu \mathrm{g}$ 3D8 scFv, respectively. All groups were treated with PBS and 3D8 scFv protein at $12 \mathrm{~h}$ intervals for 3 days. After each group was treated with PRV and 3D8 scFv, they were observed at $12 \mathrm{~h}$ intervals to determine the number of dead animals, and muscle and brain were harvested and stored at $-70^{\circ} \mathrm{C}$ for further analyses.

\section{RNA preparation and quantitative real-time reverse transcription polymerase chain reaction (RT-PCR)}

RNAs were isolated from the liver, muscle, lung, and brain tissues of C57BL/6 mice using CorezolT ${ }^{\mathrm{TM}}$ reagent (Corebio System, USA) after viral challenge (Haimov-Kochman et al., 2006). cDNA was synthesized from $5 \mu \mathrm{g}$ total RNA using random hexamers and Moloney murine leukemia virus reverse transcriptase (SuperBio). All primers were designed with the Primer 3 program (Supplementary Table S1). Expression of the PRV $\mathrm{gpD}$ transcript was evaluated via RT-PCR. Expression of C-X-C motif chemokine 10 (CXCL10) and inducible nitric oxide synthase (iNOS) (chemokine-related genes) was assayed via qRTPCR using a Rotor-Gene 3000 (Corbett Research, Australia). The relative expression of chemokine mRNAs was calculated after normalization to the GAPDH gene using the Delta $\mathrm{C}_{T}$ method (Jung et al., 2013). Each qRT-PCR was representative of data from three replicate experiments. Data represent the average of three individual experiments and error bars indicate standard errors (SEs).

\section{Immunohistochemical staining}

Representative 3- $\mu \mathrm{m}$ thick brain tissue sections were mounted on silane-coated slides for immunohistochemical analysis as described previously by Ramos-Vara (2005). Anti-PRV gpD antibody (Jeno Biotech Inc., Korea), which was used as the primary antibody $(1: 100)$ in this study, was administered for 60 min at RT. The samples were treated for color development using an iVIEW DAB Detection Kit (Ventana Medical Systems, USA).

\section{Statistical analysis}

All analyses were conducted using the GraphPAD Prism program (GraphPAD Software, USA). One-way analysis of variance and Tukey's post-hoc $t$-tests were employed for statistical analyses. Data are expressed as mean \pm SE. A $p<0.05$ was considered significant.

\section{RESULTS}

Purification of 3D8 scFv protein and catalytic affinity assay The purified $\mathrm{scFv}$ protein appeared as a single band at approximately $32 \mathrm{kDa}$ on a $10 \%$ acrylamide gel (Figs. $1 \mathrm{~A}$ and
1B). In Limulus Amebocyte Lysate (LAL) assay (PYROGENT ${ }^{\mathrm{TM}}$ 25 single tests $0.125 \mathrm{EU} / \mathrm{ml}$ sensitivity, Switzerland), the endotoxin concentration was not detected in test tubes which 3D8 scFv protein was added (Values of 3D8 scFv endotoxicity was $<0.125 \mathrm{EU} \mathrm{ml}^{-1}$ ). To investigate nuclease activity, DNA and RNA were treated with purified 3D8 ScFv protein. A catalytic affinity assay was performed dependent on reaction time. When BSA as a negative control was treated to different types of nucleic acids (dsDNA, ssDNA, dsRNA, ssRNA), any nucleic acid was not hydrolyzed (Fig. 1C). However, when ssDNA and ssRNA were incubated with endotoxin-free 3D8 scFv, ssDNA and ssRNA were degraded from $1 \mathrm{~h}$ after 3D8 scFv treatment. In contrast to ssDNA and ssRNA, dsDNA and dsRNA were gradually degraded by 3D8 ScFv as times went on. Taken together, we confirmed the catalytic activity of 3D8 scFv against all nucleotide types even though the hydrolyzing activity was slightly different among the types of nucleotides in timedependent manner.

Blood chemistry analysis for 3D8 scFv in C57BL/6 mice Blood samples were collected from mice injected with endotoxin-free 3D8 scFv and toxicity was assayed by measuring several representative parameters, followed by comparison with the samples collected from the control mice. The levels of aspartate aminotransferase and creatine phosphokinase increased significantly upon treatment with $100 \mu \mathrm{g}$ 3D8 scFv relative to the PBS treatment group used as a control. In contrast, the levels of alkaline phosphatase and glucose decreased upon treatment with $20-100 \mu \mathrm{g}$ 3D8 scFv relative to that measured in the controls (Supplementary Fig. S1 and Supplementary Table S2). Taken together, the results that i.p. injection of 20$100 \mu \mathrm{g} 3 \mathrm{D} 8 \mathrm{scFv}$ caused toxic effects; therefore, treatments of $3 \mathrm{D} 8 \mathrm{scFv}$ in all subsequent experiments were administered at concentrations well below this threshold $(5-10 \mu \mathrm{g})$.

\section{Diffusion of FITC-labeled 3D8 scFv in the organs of C57BL/6 mice and verification of 3D8 scFv delivery to target cells}

The relative quantities of endotoxin-free 3D8 scFv were measured in muscle, liver, lung, and brain tissues after the initial injection to determine if i.p. injected 3D8 scFv penetrates to target tissues. FITC-labeled 3D8 scFv protein was injected i.p. at $10 \mu \mathrm{g} / 20 \mathrm{~g}$ mouse. Total protein from each of the tissue samples was subsequently prepared at time intervals of 0,20 , $40,60,120$, and $180 \mathrm{~min}$, and the levels of FITC-3D8 scFv were measured by flow cytometry at an excitation wavelength of $494 \mathrm{~nm}$ (Fig. 2A). The 3D8 scFv protein was detected in all tissues 20 min after injection, and the protein level peaked at 60 min post-injection (20-30 fluorescence density). The protein level was maintained at a fluorescence density of approximately 15-20 for up to $120 \mathrm{~min}$ after injection. These findings demonstrate that the injected 3D8 scFv protein was transported through the bloodstream to reach target tissues, and that was stably maintained in these cells for at least a short time (Fig. 2A). This experiment was conducted using different mice, and the same results were consistently observed in all cases.

Actual penetration of 3D8 scFv was monitored in vitro using the B16F10 and C6 cell lines derived from mouse epithelia and glioma, respectively. Exogenously treated 3D8 scFv protein was detected in the cytoplasm of the cell lines approximately 48 $\mathrm{h}$ later (Fig. 2B). The results of these in vitro cell culture and in vivo mice experiments indicate that 3D8 scFv could transport to systemically each organ of C57BL/6 mice and 3D8 scFv could penetrate into cells (B16F10 and C-6). 

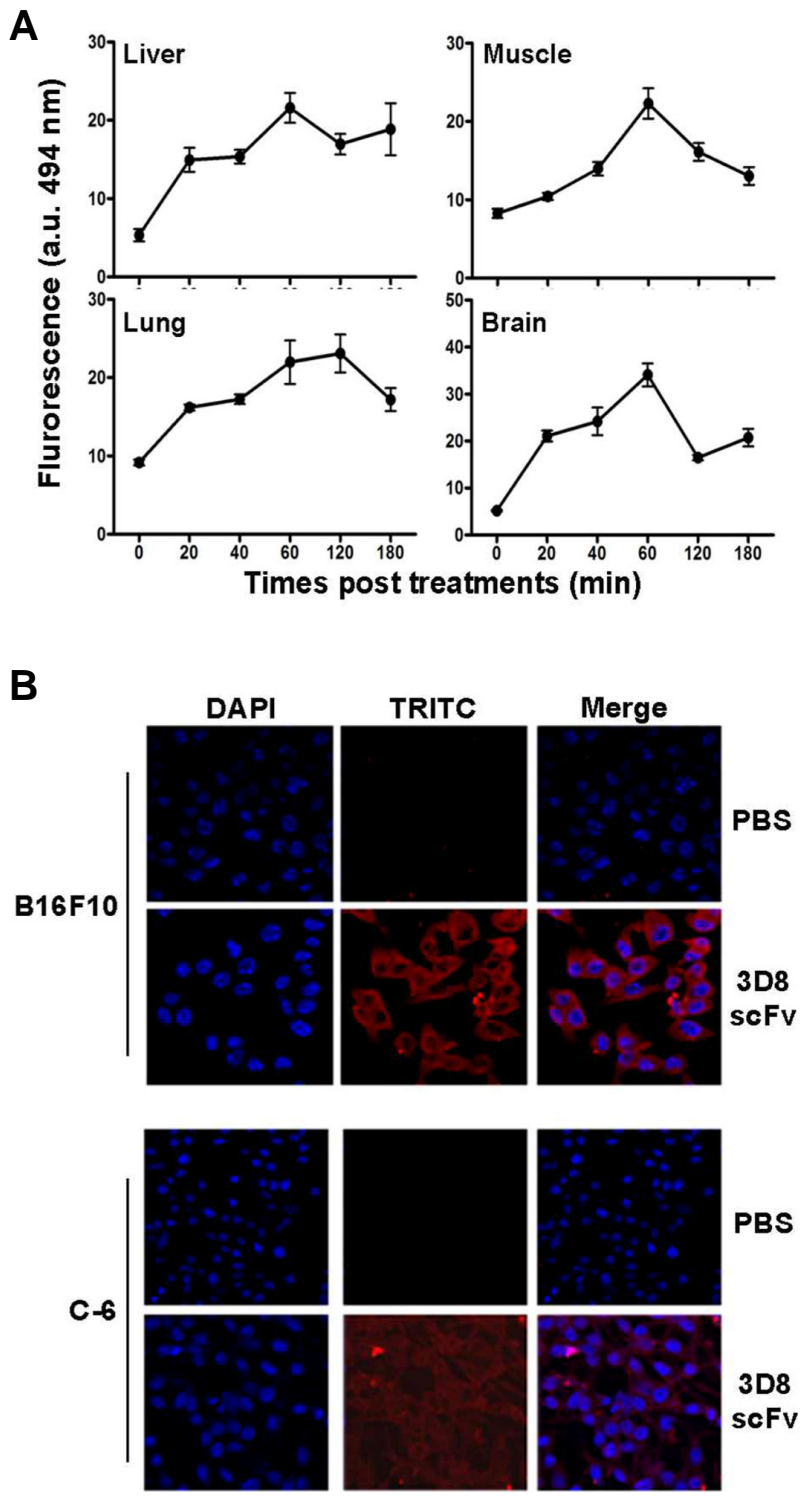

Fig. 2. The 3D8 single chain variable fragment (scFv) protein penetrates tissues (liver, muscle, lung and brain) and cells (B16F10 and C6). (A) FITC-labeled 3D8 scFv protein was injected intraperitoneally at $10 \mu \mathrm{g} / 20 \mathrm{~g}$ mouse into C57BL/6 mice. The organs (liver, muscle, lung and brain) of all mice were collected at $0,20,40,60,120$, and $180 \mathrm{~min}$, and measured at $494 \mathrm{~nm}$ via flow cytometry. Fluorescence density (a.u.: arbitrary unit) of three independent trials is shown. (B) Penetration of 3D8 scFv into cultured cells (B16F10 and C6) was evaluated by immunocytochemistry with confocal microscopy. 3D8 scFv was visualized by TRITC (rhodamine)-labeled anti-3D8 polyclonal scFv antibody. Upper panels: PBS treatment, bottom panels: 3D8 scFv treatment.

\section{D8 scFv-injected C57BL/6 mice survive exposure to PRV-YS}

The effect of 3D8 scFv as an antiviral agent against PRV in a mouse model was evaluated at protein injection concentrations of 5 and $10 \mu \mathrm{g}$ (Fig. 3A). The virus was inoculated i.m. in parallel with an i.p. injection of 3D8 scFv. The mice generally began to exhibit clinical signs of illness 3-5 days post-challenge. Approximately $57 \%$ of the group inoculated with the $L D_{50}$ of the virus without 3D8 scFv (group A) survived, whereas the groups treated with the 3D8 scFv at a concentration of $5 \mu \mathrm{g}$ (group B) or $10 \mu \mathrm{g}$ (group C) evidenced survival rates of $81 \%$ and $76 \%$, respectively, at the same lethal viral dose. Survival rate dropped significantly to $9 \%$ in the group that did not receive 3D8 scFv (group D) when inoculated with a much higher viral titer (10x $\left.\mathrm{LD}_{50}\right)$. However, the 3D8 scFv-treated groups had survival rates of $57 \%$ (5 $\mu \mathrm{g}$; group E) and 47\% (10 $\mu \mathrm{g}$; group F) (Fig. 3B). A typical symptom of PRV infection in each group is shown in Fig. 3C. Lesions in the femoral muscles of mice in group $D$ were induced as a result of viral infection, leading to death of the mice, which represented typical symptoms of the disease (Fig. $3 C)$.

\section{The 3D8 scFv protein has antiviral therapeutic effects}

Viral replication and gene and protein expression levels were measured in each group using RT-PCR and immunohistochemistry to confirm that the observed difference in survival rate after treatment with $3 \mathrm{D} 8 \mathrm{scFv}$ was the result of reduced viral pathogenicity. The RT-PCR analyses conducted on RNA isolated from the groups described in Fig. 4 showed that the viral transcript glycoprotein $\mathrm{D}(\mathrm{gpD})$ was readily detected in the RNA samples isolated from dead mice in the groups treated with 3D8 scFv (groups $\mathrm{E}$ and F). However, no gpD transcripts were detected in samples isolated from survivors of the same groups (Fig. 4A). Similarly, the gpD protein, as measured by immunohistochemistry, was detectable only in the mice that died from viral infection, whereas the protein was not detected in any of the surviving mice, regardless of their 3D8 scFv treatment status (Fig. 4B).

The antiviral effect is linked to 3D8 scFv catalytic activity The expression levels of genes involved in the inflammation response were assessed via qRT-PCR with RNA samples obtained from the groups described in Fig. $3 A$ to determine whether the antiviral mechanisms of 3D8 scFv include any indirect effects such as triggering of endogenous antiviral responses. As demonstrated in Fig. 5, the levels of two transcripts associated with the inflammation response, CXCL10 and iNOS, were detected at elevated levels in the PRV-infected samples (groups D-F). However, no significant differences in the expression levels of these transcripts were observed between the survivors and dead among these groups, thereby confirming the absence of any indirect effect of the antiviral mechanism exploited by 3D8 scFv (Figs. 5A and 5B).

\section{DISCUSSION}

A number of studies have developed effective antiviral agents, including DNA vaccines, viral DNA replication inhibitors, and viral neuraminidase inhibitors (Dory et al., 2005; Erlich and Mills, 1985; Fox et al., 2006; Koziel and Peters, 2007; Laskin, 1984; Le et al., 2005). 3D8 scFv can be distinguished from these antiviral agents, as it can digest the viral genome itself with no sequence specificity. Thus, this agent may be the vanguard of a new paradigm in antiviral therapeutics, in which the host is protected against a broad spectrum of viruses, including DNA and RNA viruses. Using the findings of prior studies, we determined that 3D8 scFv induces a series of antiviral effects against RNA viral genomes at the cellular level; thus, implicating $3 \mathrm{D} 8 \mathrm{scFv}$ as a potentially valuable antiviral therapeutic (Jun et al., 2010). In this study, we extended our ongoing research by 


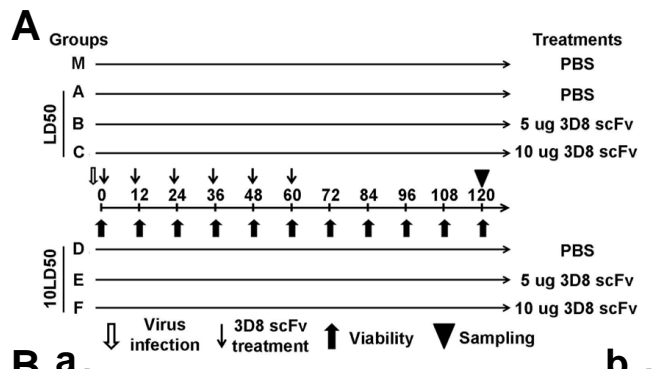

\section{C}

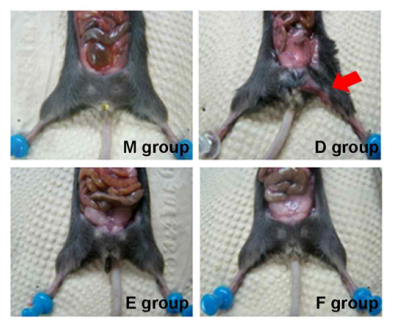

$B$ a
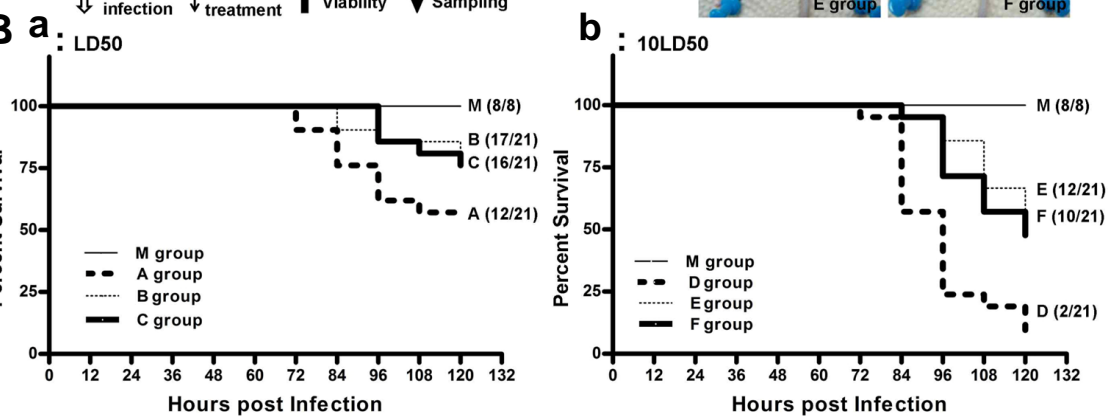

Fig. 3. $3 \mathrm{D} 8 \mathrm{scFv}$-treated $\mathrm{C} 57 \mathrm{BL} / 6$ mice demonstrate high survival rates against pseudorabies virus (PRV) infection. (A) Schematic diagram for PRV and 3D8 scFv challenge protocol. M: Mock-treated group; A, B, and C: Groups challenged with $P R V$ at $L D_{50} ; D-F$ : Groups challenged with $10 \mathrm{LD}_{50}$. Different amounts of 3D8 scFv administered to each group, indicated at right. (B) Viability of mouse groups showing correlation with the amount of 3D8 scFv treatment. (a) Effect of $3 \mathrm{D} 8 \mathrm{scFv}$ on the survival rate of $\mathrm{LD}_{50}$ PRV infection groups (A: PBS, B: $5 \mu \mathrm{g}$ 3D8 scFv, C: $10 \mu \mathrm{g}$ 3D8 scFv) and (b). Effect of 3D8 scFv on the survival rate of $10 L_{50}$ PRV infection groups (D: PBS, E: $5 \mu \mathrm{g}$ 3D8 scFv, F: $10 \mu \mathrm{g}$ 3D8 scFv). Results obtained from 21 mice per group (M group: eight mice) for up to 5 days after PRV infection. (C) Varying degrees of

symptom de- velopment after PRV infection in the mice groups, as described in (A). Dissection picture of group D shows the most severe dermatitis formation, as indicated by red arrows.

$\boldsymbol{A}$

\section{a : $E$ and $F$ live mice}

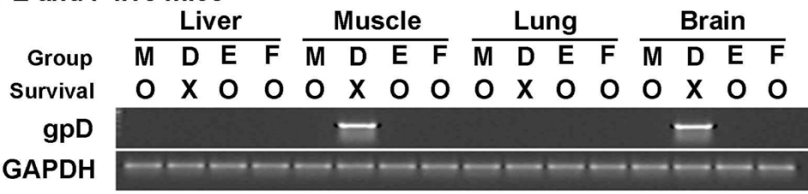

b : $E$ and $F$ dead mice

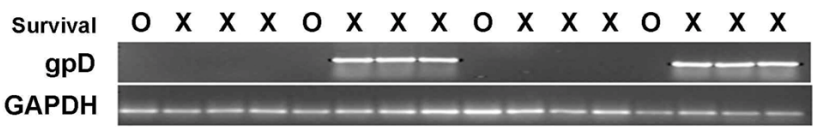

B

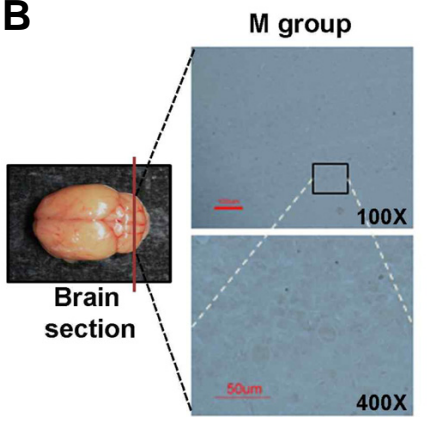

D group

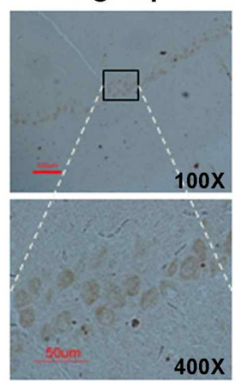

E group

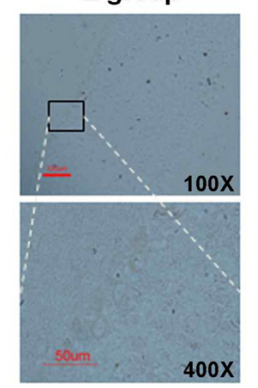

Fig. 4. $3 \mathrm{D} 8 \mathrm{scFv}$ protein exerts antiviral therapeutic effects. (A) Absence of viral transcript expression (pseudorabies virus [PRV] $\mathrm{gpD}$ ) in mice treated with the 3D8 scFv, as shown by reverse transcription-polymerase chain reaction analysis results. RNA samples from various organs (liver, muscle, lung, and brain) of different mouse groups were analyzed for PRV gpD expression. M: Mock-treated group; D challenged with $10 L^{2} D_{50} P R V$ followed by PBS treatment; $E$ challenged with $10 L_{50}$ PRV followed by $5 \mu \mathrm{g}$ 3D8 scFv treatment; $F$ challenged with $10 \mathrm{LD}_{50}$ PRV followed by $10 \mu \mathrm{g}$ 3D8 scFv treatment. Both surviving $(\mathrm{O})$ and dead $(\mathrm{X})$ mice were analyzed for PRV gpD expression in each group. Data from surviving mice in groups $E$ and $F$ are provided in (a) and data from the dead mice in groups $E$ and $F$ are shown in (b). (B) Absence of viral protein expression (PRV gpD) in mice treated with 3D8 scFv as detected via immunohistochemistry. Brain cerebellum tissues of $M, D$, and $E$ mouse groups were stained with anti-PRV monoclonal antibody visualized with biotinylated anti-mouse secondary antibody followed by DAB staining. Magnification: $100 \times$ for upper panel, $400 \times$ for bottom panel. Presence of PRV gpD protein (shown as brown staining) is detectable only in the Purkinje layer cells of the group $D$ mice. demonstrating the efficacy of 3D8 scFv against PRV in a mouse model.

Brittle et al. (2004) demonstrated that PRV is transmitted through the mouse nervous tissue to reach the brain and confirmed that the Purkinje layer of the cerebellum serves as the principal pathway by which viral transmission occurs (Brittle et al., 2004; Grieco et al., 1997). As shown in Fig. 2 of our study, i.p. injected 3D8 scFv was delivered gradually to the liver, lung, muscle, and brain over time. This result corroborates previous observations made by other research groups in which an i.p. injection delivered docetaxel much more efficiently to ascite fluid and abdominal tissues of mice than an intranasal injection (Shimada et al., 2005). The cTfRMAB-scFv protein employed as a monoclonal antibody against the transferrin receptor readily targets brain tissues and crosses the blood brain barrier after i.p. injection (Boado et al., 2010). Therefore, transport of a scFv 

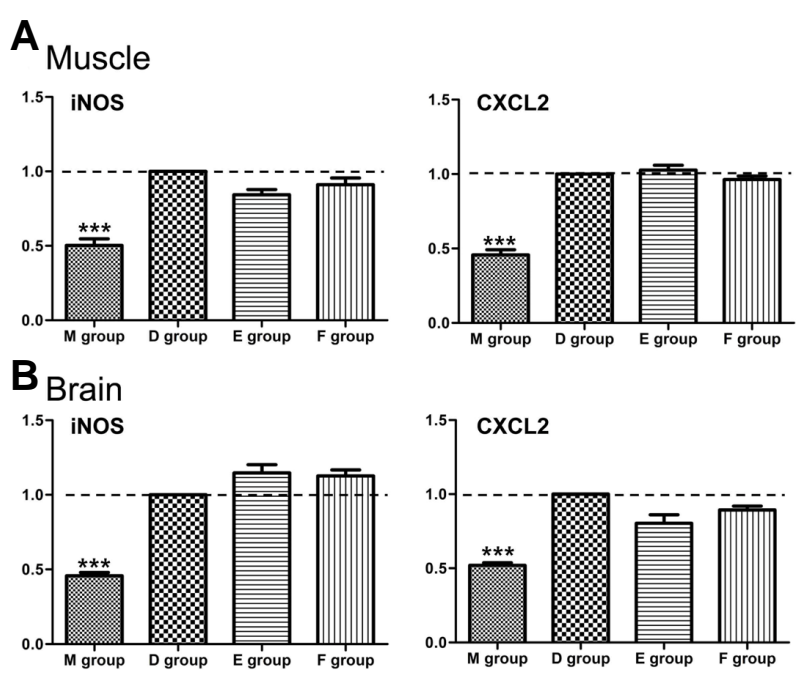

Fig. 5. The antiviral resistance observed in the 3D8 scFv-treated mice is not dependent on chemokines. Quantitative real-time reverse transcription-polymerase chain reaction analysis of the chemokinerelated genes in the pseudorabies virus (PRV)-infected mice treated with (groups $E$ and F) or without 3D8 scFv (group D). (A) Inducible nitric oxide synthase (iNOS) and C-X-C motif chemokine 10 (CXCL10) mRNA expression in muscle of mice following PRV infection. (B) iNOS and CXCL10 mRNA expression in the brains of mice following PRV infection. Data are mean \pm standard error. ${ }^{* * *}$ Significantly different from group $D$ at $p<0.001$ (one-way analysis of variance and Tukey's post hoc $t$-test). M: Mock-treated group; $D$ challenged with $10 L D_{50}$ PRV followed by PBS treatment; $E$ challenged with $10 L D_{50}$ PRV followed by treatment with $5 \mu \mathrm{g}$ of 3D8 scFv; F challenged with $10 \mathrm{LD}_{50}$ PRV followed by $10 \mu \mathrm{g}$ of $3 \mathrm{D} 8 \mathrm{scFv}$ treatment.

fragment, such as 3D8 scFv, to different organs and tissues subsequent to i.p. injection is likely to reflect a more general phenomenon.

The effect of 3D8 ScFv on viral titer was evaluated in different mouse organs infected with PRV alone or coupled with 3D8
scFv treatments. In the groups with high survival rates following viral infection due to the effects of 3D8 scFv (e.g., group $E$ and $F$ ), no viral protein PRV-gpD was detectable in any of the organs of the surviving mice. In contrast, the viral protein was detected in dead individuals of the same group, but only in muscle and brain and not in liver or lung tissues. These results suggest that the injected 3D8 scFv was transmitted to virtually all parts of the mouse, conferring resistance against the virus in all organs examined herein. This finding also reflects that transmission of PRV principally occurs through the nervous system, and little viral replication actually occurs within the liver and lung. The finding of abundant PRV within the muscle tissues is probably attributable to the initial i.m. introduction of the virus. Our observed path of PRV transmission is consistent with the findings by Breshears et al. (2005), in which Saimiriine herpesvirus 1 infected via i.m. injection translocated to the central nervous system.

The expression levels of the genes involved in the inflammation pathway were monitored to determine whether the viral genome was destroyed directly by the nuclease activity of 3D8 scFv, or indirectly by turning on the endogenous antiviral responses of the host cell. Tight correlations between induction of the genes involved in the inflammation response and increased resistance against viral infection have been reported in a number of studies. iNOS and CXCL10 transcript levels are maintained at high levels in cells infected with several viruses, including herpes simplex virus and the influenza virus (Akaike et al., 1996; Daigle and Carr, 1998; Haas et al., 1999; Hsieh et al., 2006; Su et al., 1996). Therefore, we assayed the levels of iNOS and CXCL10 transcripts in cells inoculated with PRV; thus, allowing us to determine whether treatment with 3D8 scFv altered the expression levels of these transcripts in these cells. As shown in Fig. 5, although PRV-infected cells generally showed much higher levels of these transcripts relative to controls, 3D8 scFv treatment resulted in no additional changes in the transcript levels in virally-infected cells. Also, when we treated the inhibitor of type I interferon receptor as a mediator of intrinsic antiviral activity to Hantaan virus (HTNV)-infected HeLa and 3D8 scFv transgenic cells, no differences were observed between presence or absence of inhibitor in 3D8 scFv transgenic cells (data not shown). Thereby, it is demonstrate that the observed antiviral effects of 3D8 scFv were induced directly by
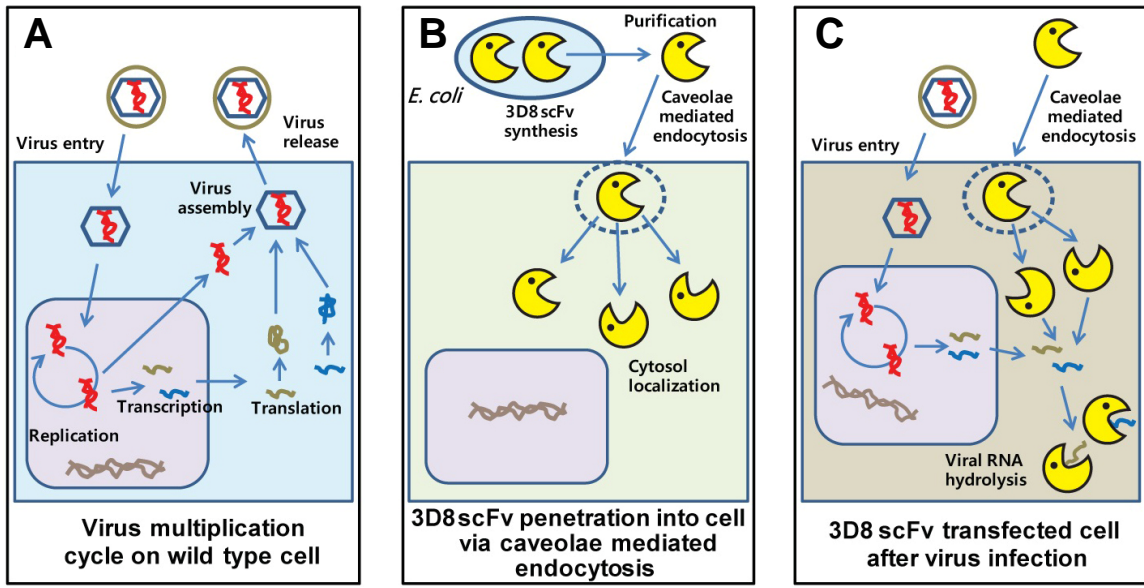

Fig. 6. Antiviral mechanism by $3 \mathrm{D} 8$ scFv protein. (A) This model shows the pseudorabies virus (PRV) replication cycle. Entry begins with attachment or binding of the virus particle to the cell surface. The viral DNA enclosed by nucleocapsid delivers into nucleus. Viral DNA synthesis begins after the expression of the beta proteins and the temporal program of viral gene expression ends with the appearance of the gamma or late proteins, which constitute the structural proteins of the virus. Finally, the virus initiates a lytic cycle. (B) Mice were injected intraperitoneally with purified 3D8 scFv protein. The treated 3D8 scFv proteins penetrate the cell via a caveolae

-mediated endocytosis mechanism. Consequentially, 3D8 scFv proteins are localized in cytoplasm. (C) 3D8 scFv proteins localized in cytoplasm degrade viral RNA by RNase activity and inhibit the expression of the viral proteins. The RNase activity of 3D8 scFv exhibits the therapeutic effects against PRV infection. 
the activities of 3D8 scFv itself.

In conclusion, as an extension of our previous studies in which the antiviral effect of 3D8 scFv was demonstrated by in vitro experiments, we further showed that nuclease activity of 3D8 scFv could be effectively employed in vivo as an antiviral therapeutic agent using the mouse model system. Purified 3D8 scFv protein from $E$. coli was i.p. injected into PRV infected mice and localized to the cytoplasm by caveolae-mediated endocytosis (Jun et al., 2010). Then, the PRV transcriptome was degraded by 3D8 scFv with intrinsic RNase activity in cytoplasm (Fig. 6). The finding that 3D8 scFv nuclease activity acts on both DNA and RNA demonstrates the utility of 3D8 scFv as a multifaceted, broad-spectrum antiviral agent that works against DNA and RNA viruses.

Note: Supplementary information is available on the Molecules and Cells website (www.molcells.org).

\section{ACKNOWLEDGMENTS}

We are indebted to Dr. Jae-Young Song and his group at the National Veterinary Research and Quarantine Service (NVRQS). This study was supported by the Agenda (no. PJ0102012015) from the Rural Development Administration in Korea and the Korea Institute of Ocean Science and Technology project (no. PE99154) of Korea.

\section{REFERENCES}

Akaike, T., Noguchi, Y., ljiri, S., Setoguchi, K., Suga, M., Zheng, Y.M. Dietzschold, B., and Maeda, H. (1996). Pathogenesis of influenza virus-induced pneumonia: involvement of both nitric oxide and oxygen radicals. Proc. Natl. Acad. Sci. USA 93, 24482453.

Banks, P.R., and Paquette, D.M. (1995). Comparison of three common amine reactive fluorescent probes used for conjugation to biomolecules by capillary zone electrophoresis. Bioconjug. Chem. 6, 447-458.

Boado, R.J., Zhou, Q.H., Lu, J.Z., Hui, E.K., and Pardridge, W.M. (2010). Pharmacokinetics and brain uptake of a genetically engineered bifunctional fusion antibody targeting the mouse transferrin receptor. Mol. Pharm. 7, 237-244.

Breshears, M.A., Eberle, R., and Ritchey, J.W. (2005). Temporal progression of viral replication and gross and histological lesions in Balb/c mice inoculated epidermally with Saimirine herpesvirus 1 (SaHV-1). J. Comp. Pathol. 133, 103-113.

Brinkley, M. (1992). A brief survey of methods for preparing protein conjugates with dyes, haptens, and cross-linking reagents. Bioconjug. Chem. 3, 2-13.

Brittle, E.E., Reynolds, A.E., and Enquist, L.W. (2004). Two modes of pseudorabies virus neuroinvasion and lethality in mice. J. Virol. $78,12951-12963$.

Daigle, J., and Carr, D.J. (1998). Androstenediol antagonizes herpes simplex virus type 1-induced encephalitis through the augmentation of type I IFN production. J. Immunol. 160, 30603066.

Dory, D., Torche, A.M., Beven, V., Blanchard, P., Loizel, C., Cariolet, R., and Jestin, A. (2005). Effective protection of pigs against lethal Pseudorabies virus infection after a single injection of lowdose Sindbis-derived plasmids encoding $\mathrm{PrV} \mathrm{gB}, \mathrm{gC}$ and $\mathrm{gD}$ glycoproteins. Vaccine 23, 3483-3491.

Enquist, L.W. (1999). Life beyond eradication: veterinary viruses in basic science. Arch. Virol. Suppl. 15, 87-109.

Erlich, K.S., and Mills, J. (1985). Chemotherapy for herpes simplex virus infections. West J. Med. 143, 648-655.

Ferraris, O., and Lina, B. (2008). Mutations of neuraminidase implicated in neuraminidase inhibitors resistance. J. Clin. Virol. 41, 13-19.

Field, H.J., and Hill, T.J. (1974). The pathogenesis of pseudorabies in mice following peripheral inoculation. J. Gen. Virol. 23, 145157.

Fox, Z., Dragsted, U.B., Gerstoft, J., Phillips, A.N., Kjaer, J.,
Mathiesen, L., Youle, M., Katlama, C., Hill, A., Bruun, J.N., et al. (2006). A randomized trial to evaluate continuation versus discontinuation of lamivudine in individuals failing a lamivudinecontaining regimen: the COLATE trial. Antivir. Ther. 11, 761-770.

Gerdts, V., Jons, A., Makoschey, B., Visser, N., and Mettenleiter, T.C. (1997). Protection of pigs against Aujeszky's disease by DNA vaccination. J. Gen. Virol. 78 (Pt 9), 2139-2146.

Grieco, V., Gelmetti, D., Finazzi, G., Brocchi, E., and Finazzi, M. (1997). Immunohistologic diagnosis of pseudorabies (Aujeszky's disease) using monoclonal antibodies. J. Vet. Diagn. Invest. 9, 326-328.

Haas, J., Meyding-Lamade, U., Fath, A., Stingele, K., StorchHagenlocher, B., and Wildemann, B. (1999). Acyclovir treatment of experimentally induced herpes simplex virus encephalitis: monitoring the changes in immunologic NO synthase expression and viral load within brain tissue of SJL mice. Neurosci. Lett. 264, 129-132.

Haimov-Kochman, R., Fisher, S.J., and Winn, V.D. (2006). Modification of the standard Trizol-based technique improves the integrity of RNA isolated from RNase-rich placental tissue. Clin. Chem. 52, 159-160.

Hoang, P.M., Cho, S., Kim, K.E., Byun, S.J., Lee, T.K., and Lee, S. (2014). Development of Lactobacillus paracasei harboring nucleic acid-hydrolyzing 3D8 scFv as a preventive probiotic against murine norovirus infection. Appl. Microbiol. Biotechnol. 99, 27932803.

Hong, W., Xiao, S., Zhou, R., Fang, L., He, Q., Wu, B., Zhou, F., and Chen, H. (2002). Protection induced by intramuscular immunization with DNA vaccines of pseudorabies in mice, rabbits and piglets. Vaccine 20,1205-1214.

Hsieh, M.F., Lai, S.L., Chen, J.P., Sung, J.M., Lin, Y.L., Wu-Hsieh, B.A., Gerard, C., Luster, A., and Liao, F. (2006). Both CXCR3 and $\mathrm{CXCL} 10 / \mathrm{IFN}$-inducible protein 10 are required for resistance to primary infection by dengue virus. J. Immunol. 177, 1855-1863.

Jang, J., Jeong, J., Jun, H., Lee, S., Kim, J., Kim, Y., and Kwon, M. (2009). A nucleic acid-hydrolyzing antibody penetrates into cells via caveolae-mediated endocytosis, localizes in the cytosol and exhibits cytotoxicity. Cell. Mol. Life Sci. 66, 1985-1997.

Jun, H.R., Pham, C.D., Lim, S.I., Lee, S.C., Kim, Y.S., Park, S., and Kwon, M.H. (2010). An RNA-hydrolyzing recombinant antibody exhibits an antiviral activity against classical swine fever virus. Biochem. Biophys. Res. Commun. 395, 484-489.

Jung, Y.D., Ha, H.S., Park, S.J., Oh, K.B., Im, G.S., Kim, T.H., Seong, H.H., and Kim, H.S. (2013). Identification and promoter analysis of PERV LTR subtypes in NIH-miniature pig. Mol. Cells 35, 99-105.

Kim, Y.R., Kim, J.S., Lee, S.H., Lee, W.R., Sohn, J.N., Chung, Y.C., Shim, H.K., Lee, S.C., Kwon, M.H., and Kim, Y.S. (2006). Heavy and light chain variable single domains of an anti-DNA binding antibody hydrolyze both double- and single-stranded DNAs without sequence specificity. J. Biol. Chem. 281, 15287-15295.

Koziel, M.J., and Peters, M.G. (2007). Viral hepatitis in HIV infection. N Engl. J. Med. 356, 1445-1454.

Laskin, O.L. (1984). Acyclovir. Pharmacology and clinical experience. Arch. Intern. Med. 144, 1241-1246.

Le, Q.M., Kiso, M., Someya, K., Sakai, Y.T., Nguyen, T.H., Nguyen, K.H., Pham, N.D., Ngyen, H.H., Yamada, S., Muramoto, Y., et al. (2005). Avian flu: isolation of drug-resistant H5N1 virus. Nature 437, 1108.

Lee, G., Shim, H.K., Kwon, M.H., Son, S.H., Kim, K.Y., Park, E.Y., Lee, T.K., Lee, W.R., Auh, C.K., and Kim, D. (2013a). A nucleic acid hydrolyzing recombinant antibody confers resistance to curtovirus infection in tobacco. Plant Cell Tissue Organ Culture 115, 179-187.

Lee, G., Shim, H.K., Kwon, M.H., Son, S.H., Kim, K.Y., Park, E.Y., Yang, J.K., Lee, T.K., Auh, C.K., and Kim, D. (2013b). RNA virus accumulation is inhibited by ribonuclease activity of 3D8 ScFv in transgenic Nicotiana tabacum. Plant Cell Tissue Organ Culture 115, 189-197.

Lee, G., Yu, J., Cho, S., Byun, S.J., Kim, D.H., Lee, T.K., Kwon, M.H., and Lee, S. (2014). A nucleic-acid hydrolyzing single chain antibody confers resistance to DNA virus infection in hela cells and C57BL/6 mice. PLoS Pathog. 10, e1004208.

McCracken, R.M., McFerran, J.B., and Dow, C. (1973). The neural spread of pseudorabies virus in calves. J. Gen. Virol. 20, 17-28. 
Morfin, F., and Thouvenot, D. (2003). Herpes simplex virus resistance to antiviral drugs. J. Clin. Virol. 26, 29-37.

Ono, E., Yoshino, S., Amagai, K., Taharaguchi, S., Kimura, C., Morimoto, J., Inobe, M., Uenishi, T., and Uede, T. (2004). Enhanced resistance to herpes simplex virus type 1 infection in transgenic mice expressing a soluble form of herpesvirus entry mediator. Virology 320, 267-275.

Pensaert, M., and Kluge, J. (1989). Virus infections of porcines (Amsterdam, The Netherlands: Elsevier Science Publishers BV). Ramos-Vara, J.A. (2005). Technical aspects of immunohistochemistry. Vet. Pathol. 42, 405-426.

Shimada, T., Nomura, M., Yokogawa, K., Endo, Y., Sasaki, T., Miyamoto, K., and Yonemura, Y. (2005). Pharmacokinetic advantage of intraperitoneal injection of docetaxel in the treat- ment for peritoneal dissemination of cancer in mice. J. Pharm. Pharmacol. 57, 177-181.

Stegeman, A., de Jong, M.C., van Nes, A., and Bouma, A. (1997) Dynamics of pseudorabies virus infections in vaccinated pig populations: a review. Vet. Q 19, 117-122.

Su, Y.H., Yan, X.T., Oakes, J.E., and Lausch, R.N. (1996). Protective antibody therapy is associated with reduced chemokine transcripts in herpes simplex virus type 1 corneal infection. J. Virol. 70, 1277-1281.

Yoon, H.A., Han, Y.W., Aleyas, A.G., George, J.A., Kim, S.J., Kim, H.K., Song, H.J., Cho, J.G., and Eo, S.K. (2008). Protective immunity induced by systemic and mucosal delivery of DNA vaccine expressing glycoprotein B of pseudorabies virus. J. Microbiol. Biotechnol. 18, 591-599. 